\title{
Application of Decision Analytic Model in Health Economic Evaluation: Smoking Cessation Cases
}

\author{
Septiara Putri ${ }^{1}$ \\ Contact: septiara.putri@cheps.or.id \\ Submitted on July 18, 2016. Reviewed July 25, 2016, and accepted on July 25, 2016
}

\begin{abstract}
Health economic evaluation that encompasses decision analytic model is a beneficial approach for assisting decision maker to choose the best health intervention for patients. Decision analytic model has been increasingly applied in health economic evaluation. This mathematical approach is mostly used for conducting cost-effectiveness of healthcare interventions.
\end{abstract}

Decision tree and Markov model has been widely applied in the past 20 years. Decision tree is the simplest form of decision model that drawn by the series of branches and clear pathways. Meanwhile, Markov model is one of the powerful approaches that employ stochastic process in health economic evaluation. This paper describes the applications of those two models in tobacco cessations, specifically for pharmacological interventions.

First, decision tree for cost-effectiveness of smoking cessation program with pharmacist and therapies interventions compared to no program or self-aid cessation. Second, the application of Markov model estimates cost-effectiveness of veranicline, in comparison to bupropion. Markov model is constructed with morbidity and mortality states that consists of: well/no morbidities, lung cancer, COPD, stroke, myocardial infarction, and dead. This paper provides step by step of populating and constructing the model-with some modification of data. Several sections discuss the understanding of transition probabilities, costs data, cohort simulation, and the role of sensitivity analysis. Other models, despite deterministic approach, probabilistic approach are also reviewed.

Both of models had both advantages and limitation that analysts should be aware of. Translating the 'real world' to mathematical model yields beneficial and insightful information for analysts. In addition, it could fulfill the need of evidence-based policy by decision maker. From simulation, the model may easy to be replicated-with appropriate context to generate evidence related health and costs.

Key Words :

Smoking Cessation. Tobacco Control. Modeling. Markov Model. Decision Tree. Indonesia

1 Research Assistant (Center for Health Economic and Policy Studies UI) 


\section{Introduction}

Health economic evaluation is a general framework to aid decision maker with best available evidence in terms of priority setting and choosing particular health technologies (Shiell et al. 2002). This is one of pivotal aspects for decision making that commonly related to funding, reimbursement or regulatory of health intervention, with increasing challenges of finite resources available (Morris et al. 2007; Hjelmgren et al. 2001).

Decision analytic model can be used in health economic evaluation; it is widely applied to estimate cost-effectiveness of healthcare interventions (Sox et al. 1988; Buxton et al.1997; Hunnink et al. 2001). This essential technique is defined as a systematic approach on synthesizing information from multiple sources and applying mathematical relationship to answer decision problems, allowing its variability and uncertainty. Expected costs and possible consequences of all available options are compared (Briggs et al. 2006; Gray et al. 2010). In particular situation, economic evaluation is increasingly conducted alongside clinical trial. However, the results from this economic evaluation do not always able to inform decision related reimbursement (Sculpher et al. 2006). Decision analytic model therefore can be beneficial to resolve the limitations in clinical trial.

Generally, there are several circumstances when decision analytic modeling in health economic evaluation is very useful. First, when Randomized Clinical Trials (RCTs) could not be undertaken due to costs, time, or other reasons. Hence, modeling techniques can be applied by synthesizing all relevant information from various sources, not only from primary data (Karnon and Brown, 1998). Second, RCTs remain crucial for efficacy evidence. However, it might not compare all relevant alternatives and provide appropriate time horizon. In health economic evaluation, time horizon should be long enough to capture the magnitude of costs and benefits among alternatives. (Drummond et al., 2005; Briggs et al., 2006). Third, final endpoints evidence is difficult to be achieved, for instance: mortality and long-term morbidity. It is related to short time horizon in clinical trials, mostly RCTs are concluded up to intermediate endpoints. Furthermore, by developing a model, the results can be extended (Briggs et al., 2006). Moreover, Sculpher et al. (1997) explained four major stages in economic evaluation process, and decision analytic model assigned prominent role in each of those steps. Therefore, decision analytic model is able to sufficiently answer the resource allocation question, while all information about cost and consequences become barriers in primary clinical research such as RCTs. As powerful tool in economic evaluation, modeling is structuring evidence both clinical and economics outcomes, simplifying representation of reality. It thus beneficial to generate relevant evidence that highly required by decision makers.

There are some alternatives modeling approach that can be used for estimating cost-effectiveness of health interventions. For example: dynamic models, discrete event simulations (DES), and patient level simulations (Petrou and Gray, 2011). However, this paper only discusses decision tree and Markov model application, since these two models are frequently applied in economic evaluation (Buxton et al., 1997; Karnon and Brown 1998). Author discusses the example of tobacco program as well as pharmacological therapies in tobacco cessation, in this example: veranicline, bupropion. The costs and outcomes value have been determined as instances. Models in this paper are structured by referring from previous studies, indeed with some modifications. Author will frequently use terms Cost-effectiveness Analysis (CEA) and Cost Utility Analysis(CUA). Illustrations of these methods can be found in publication by Drummond et al. (2005). This paper is divided into several sections that cover stages on designing and conducting simple decision tree 
and Markov model for pharmacological interventions in smoking cessation.

This paper neither demonstrates modeling choice nor provides ideal good structure that should be followed on tobacco cessation cases. Models are characterized depend on research or policy question, modeler, and decision maker consideration, not only technically applying mathematical operations. It should be noted that this paper focuses on outlining the basic theory and aim to familiarize unexposed audience with basics modeling applications as abstractions of reality.

\section{Decision rules}

Before going to further parts, this section covers the decision rules and general rationale of conducting CEA/CUA for making sense the final resources after applying decision models. Decision makers in health sector often face a dilemma for choosing health intervention. New treatment/ health technology is often more effective than current/standard treatment, however it is inevitably more expensive. Health economists are interested in considering of "how much do we get the benefit for additional cost or the money spent?" Value for money of new intervention furthermore is summarized by Incremental Cost-Effectiveness Ratio (ICER) (Owens, 1998; Jefferson, 2000; Cohen and Reynolds, 2008).

The concept of cost, effect, effectiveness and its linkage to ICER concepts are divided into four main rules (O’Brien et al. 1994; Briggs and Tambour 1998). From two therapies that are compared, one new treatment and a comparator or familiarly named as existing treatment represented by "A" and "B", respectively. Mean of treatment's costs denoted by $\mu \_\mathrm{c} A$ and $\mu$ cB. Similarly, effects are denoted by $\mu_{-}$eA and $\mu_{-}$eB. It describes below:

1. $\quad \mu \_\mathrm{cA}-\mu \_\mathrm{cB}<0 ; \mu \_\mathrm{eA}-\mu \_\mathrm{eB}>0 ;$ dominance, accept new treatment

2. $\quad \mu \_\mathrm{cA}-\mu_{-} \mathrm{cB}>0 ; \mu \_\mathrm{eA}-\mu_{-} \mathrm{eB}<0 ;$ dominance, reject new treatment

3. $\mu \_\mathrm{cA}-\mu \_\mathrm{cB}>0 ; \mu \_\mathrm{eA}-\mu \_\mathrm{eB}>0$; trade-off (consider magnitude of ratio of differences in cost to differences in effect; or additional costs relative to additional effectiveness)

4. $\quad \mu \_c A-\mu \_c B<0 ; \mu \_$eA - $\mu \_$eB $<0$; trade-off (consider magnitude of ratio of differences in cost to differences in effect, cost-saving to it reduced effectiveness)

Those four points are in relation to four quadrants of cost-effectiveness plane. It is commonly used for presenting cost-effectiveness results that described and discussed in almost economic evaluation results. The condition number 1 demonstrates that we accept new treatment when it is more effective and less costly compared to existing treatment. In contrast, condition number 2, when new treatment is more costly and less effective, so then clearly we reject it. However, when a new treatment is more effective but also it is more expensive, then judgment should be made. This is the condition when ICER is playing its role, providing the summary of cost-effectiveness of new intervention compared to current intervention/treatment(s) with equation:

$$
\operatorname{ICER}=\left(\mu_{\mathrm{cA}}-\mu_{\mathrm{cB}}\right) /\left(\mu_{\mathrm{eA}}-\mu_{\mathrm{cB}}\right)=\mu_{\Delta \mathrm{c}} / \mu_{\Delta \mathrm{e}}<\lambda
$$

Therefore, ICER is simply the ratio of both differences in costs and effects. The $\lambda$ (lambda) represents maximum acceptable cost-effectiveness ratio or ceiling ratio. Several countries have produced lamda to support their decision related to new health intervention (Mc Cabe et al. 2008; Grosse, 2008; Shiroiwa et al. 2010; Neumann et al. 2014). If the ICER is less than the value of $\lambda$, it is concluded that the intervention is potentially be accepted. 


\section{Defining questions}

The first step for structuring the decision model is specifying the decision problem. Population, interventions, comparators, as well as clinical and economic outcomes should be clearly identified (Briggs et al. 2006; Drummond et al. 2005; Gray et al. 2010). In this case, for instance, we will discuss modeling method for cost-effectiveness/cost-utility analysis of smoking cessation program with pharmacological therapies such as: varenicline and bupropion. In addition, perspective of evaluation should be considered, why the intervention is being evaluated, costs incurred, and for whom the result required and may support the decision. The perspectives reflect the purpose of evaluation (Byford and Raftery, 1998; Jonsson, 2009; Hjelmegren et al. 2001).

Appropriate decision model should be structured and developed by clear understanding about the problems. Several guidelines provide details of good practices in constructing the model (Weinstein et al. 2003; Roberts et al. 2012). Parameters choice, sources of data and evidence synthesis important as model input also available in standing alone publication. (Caro et al. 2010; Briggs et al. 2012). In this paper, all the data has been given and we directly head to explanation of model structure. However, author provides the schematic of design and conducting decision analytic model in figure 1. It is a complete visual illustration of decision analysis process. This illustration is summarized and modified according several publications (Briggset al. 2006; Martikainen, 2008; Gray et al. 2010; Roberts et al. 2012; Briggs et al. 2012). 


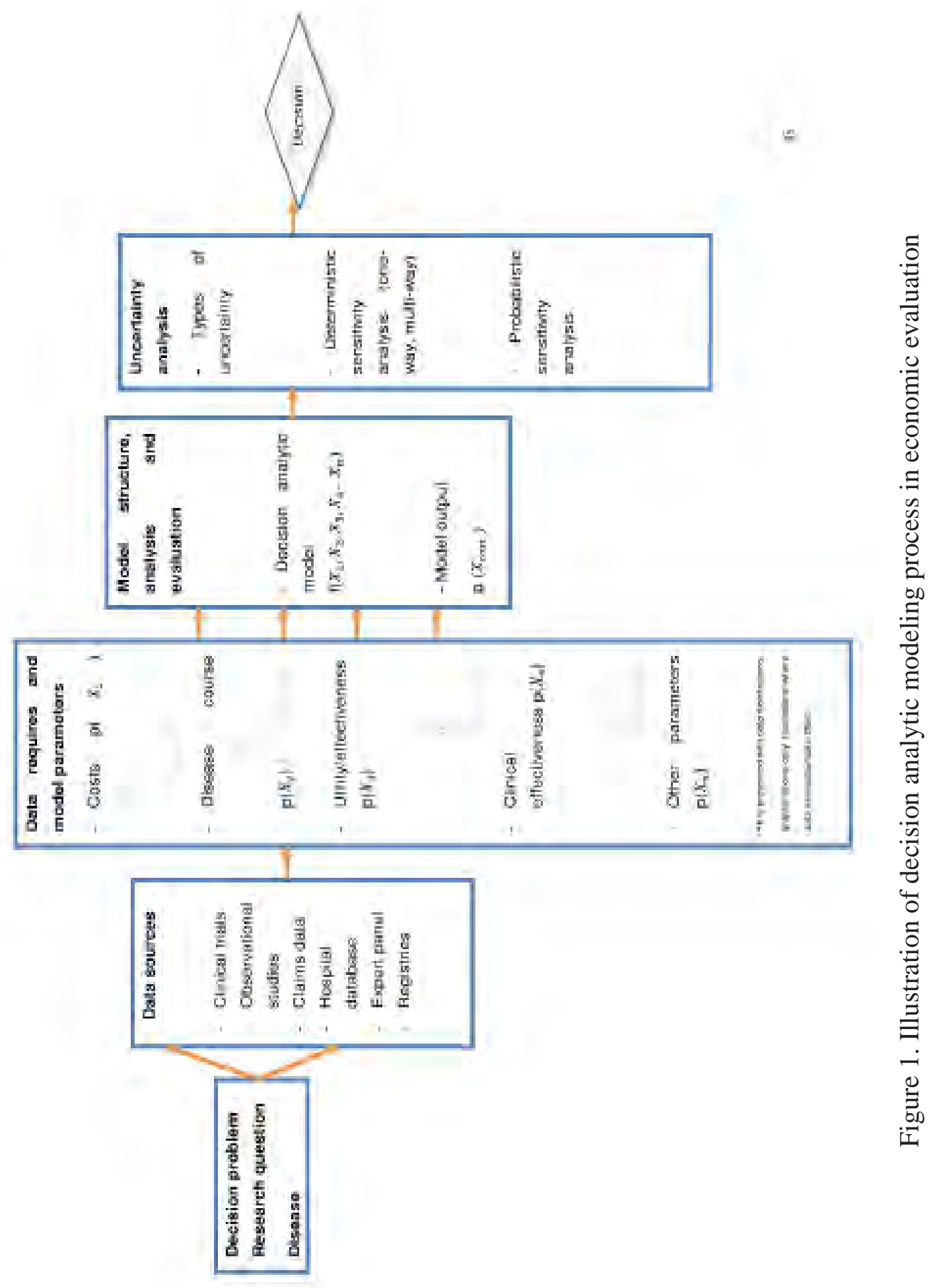




\section{Structuring and analyzing the models \\ Decision Tree}

Smoking cessation can be classified into two types behavioral therapies and pharmacotherapies. Pharmacotherapies interventions in smoking cessation can be classified as nicotine replacement therapy (NRT) and non-nicotine based medications (bupropion and varenicline) (Ruger and Razar, 2012). The first model will discuss the cost-effectiveness of community pharmacy on smoking cessation program. We will estimate the cost-effectiveness of establishing smoking cessation programs, medications compared to non-pharmacy based program.

In decision analytical modeling, the simplest form of model is decision tree (Karnon and Brown, 1998). Decision tree illustrates alternatives and its events that represent by pathways (figure 2.). There are several nodes in a decision tree. The "square" node represents the decision question, in this case pharmacological-smoking cessation program versus no program. This is the simplest scenario of a decision tree. In reality, the comparators are probably more than one, such as tobacco bans or other considerable programs. Events or morbidities in a decision tree are represented by "circular" symbols. "Triangular" or terminal nodes are placed in the end of each event pathways, where model stopped.

The set of alternatives in a decision tree should be "mutually exclusive". It means that two events could not occur simultaneously (Briggs et al. 2006, Petrou and Gray 2011). Probabilities of events and costs are incorporated in each pathway. The probability shows the proportion of patients' progression, and in each alternative it should be summed as exactly 1.

The decision tree structure is adapted from study that compares a pharmacist-managed smoking-cessation program with a self-directed quit attempt. The events from therapy strategies are modified and simplified (Figure 2.) (Tran et al. 2002; Bauld et al. 2011). A comparator or no program means there is no aid from a pharmacist in smoking cessation. The data in this model is assumed primary data. Costs incurred are cost related to intervention, with payer perspective. This model assumes that in six months, the program may generate two conditions, smokers attempt to quit smoking or remain continue to smoke, with events of their health condition, persistent cough.

There are two strategies in this model. Costs are assigned in each pathway. The effectiveness of intervention is illustrated on each event, having persistent cough or not. For instance, the probabilities 0.30 , indicating that $30 \%$ of patients who joined the program with medications attempted to quit smoking. The final result is cost per case avoided. In the end of the decision tree, the expected value of costs and outcomes are assigned, ICER therefore can be calculated. 


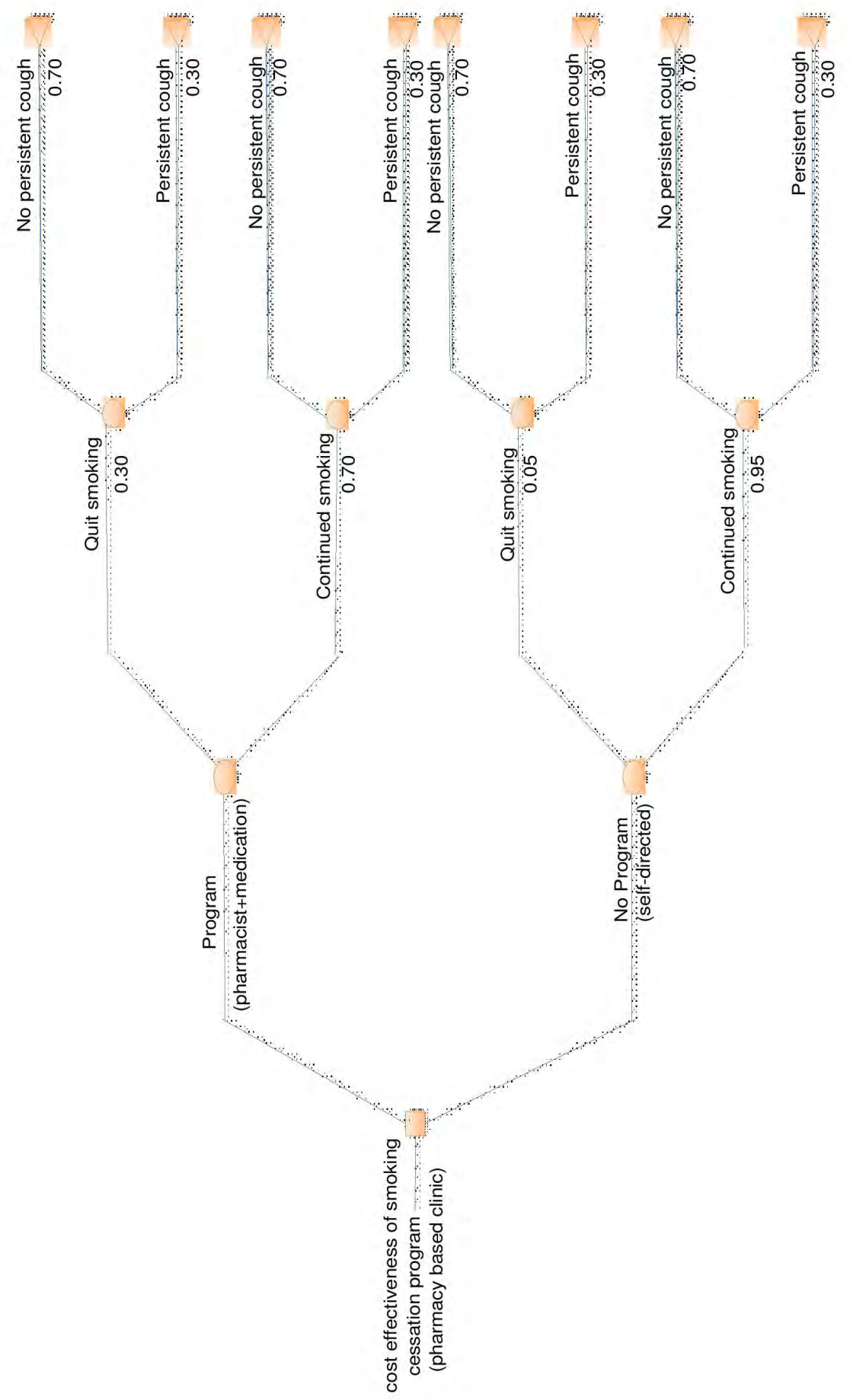

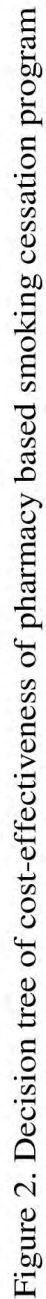


The summary of calculation is briefly presented below:

Table 1. Calculation according decision-tree

\begin{tabular}{|c|c|c|c|c|c|c|}
\hline Pathways & Costs & $\begin{array}{l}\text { Intervention } \\
\text { benefit }\end{array}$ & & \multicolumn{2}{|c|}{$\begin{array}{l}\text { Expected cost } \\
(\mathrm{a} \times \mathrm{b})\end{array}$} & Intervention benefit \\
\hline \multicolumn{7}{|l|}{ Program } \\
\hline $\mathrm{A}$ & $\$ 200$ & $0.30 \times 0.70=0.21$ & & $\$$ & 42 & $\$ 222.5 / 0.21=\$ 1,059.5$ \\
\hline B & $\$ 275$ & $0.30 \times 0.30=0.09$ & & $\$$ & 24.75 & \\
\hline $\mathrm{C}$ & $\$ 200$ & $0.70 \times 0.70=0.49$ & & $\$$ & 98 & \\
\hline $\mathrm{D}$ & $\$ 275$ & $0.70 \times 0.30=0.21$ & & $\$$ & 57.75 & \\
\hline Total & & 1.00 & & $\$$ & 222.5 & \\
\hline \multicolumn{7}{|l|}{ No program } \\
\hline $\mathrm{E}$ & $\$ 100$ & $0.05 \times 0.70=0.04$ & & $\$$ & 4 & $\$ 117.5 / 0.04=\$ 2,937.5$ \\
\hline $\mathrm{F}$ & $\$ 150$ & $0.05 \times 0.30=0.02$ & & $\$$ & 3 & \\
\hline $\mathrm{G}$ & $\$ 100$ & $0.95 \times 0.70=0.67$ & & $\$$ & 67 & \\
\hline $\mathrm{H}$ & $\$ 150$ & $0.95 \times 0.30=0.29$ & & $\$$ & 43.5 & \\
\hline Total & & & 1.00 & $\$$ & 117.5 & \\
\hline
\end{tabular}

The average cost-effectiveness ratio (ACER) is derived from expected costs per effectiveness, in this case per quit smoking with no persistent cough. We can conclude that ACER of no program is higher than providing program $\$ 2,937.5$. Furthermore, to estimate ICER, as formulated above, the ICER will be (222.5-117.5) / (0.21-0.04) resulting \$617.65 per quit attempt (with no persistent cough). To conclude whether program is potentially effective, analysts can use their acceptable maximum of cost-effectiveness ratio $(\lambda)$.

Applying decision tree allows the less complicated scenarios, simplicity, as well as transparency (Karnon and Brown, 1998; Petiti, 1999). We can build a set of alternatives with clear pathways. However, there is a limitation to deal with time dependent in economic evaluation such as applying discounting. The decision tree above looks simple because it is applying shortterm outcome and short time horizon (less than one year), with unnecessary discounting application. The smoking cases often have outcome that tend to be recursive, or patients will survive with a particular condition that may take longer than one year. In terms of recurrent events such as chronic diseases, the analysis will be more complicated. Decision tree become "bushy" and having long-complex pathways. (Karnon and Brown, 1998; Briggs et al. 2006; Petrou and Gray, 2011)

\section{Markov model}

The Markov model was first developed by Russian mathematician, Andrey Markov (1856-1922). It is a random process that encounters transition from one state to another state. This model is beneficial for ongoing events over time and risks exposure (Stahl, 2008). For instance, we will discuss lung cancer as clinical conditions that are experienced by patients, risks may change over time and Markov model can be useful for this case.

As a model alternative in health economic evaluation, the Markov model can deal with more complex decision problems and is able to be applied for longer pathways/sequences, particularly for recurrent outcomes (Stahl, 2008; Sato and Zouain, 2010). Markov state refers patients are staying in one health state and can move from their current state to another state over discrete time period. The movements among states are attributed by transition probability. Number of states in Markov model are determined depend on decision problem. Moreover, time horizon is represented by distinctive "cycles", it could be monthly or annual cycle. (Sonneberg and Beck, 1993; Briggs and Sclupher, 1998; Briggs, 2006; Nikfar, 2012). The first state in Markov process 
is commonly of well condition or without morbidity, then progression states and finally death, or familiarly known as "absorption state".

We will discuss the application of Markov model for estimating the cost-effectiveness of Veranicline compared to Bupropion. This is based on condition that Varenicline has higher effectiveness to support smoking cessation compared to another pharmacological intervention (Mahmoudi et al, 2012). Structure of this model is adapted and modified according to publication that apply BENESCO (Benefits of Smoking Cessation on Outcomes model) and Quit Benefit Model (QBM) (Hurley and Matthews 2007; Bolin et al. 2009).

The first stage in constructing Markov model after specifying decision problem is defining the states. States must represent clinical and economic effects of a disease. In this model, states consist of: no-morbidity/well, COPD (Chronic Obstructive Pulmonary Disease), lung cancer, stroke and MI (Myocardial Infarction). Four states in progressive states assume that model will be simulated for 20 years, and post-morbidities state transition probability assumed similar for first and second year onwards. The Markov model is presented in Figure 3, and to translate and to understand the transition probabilities, author provide the transition matrix on table 2 .

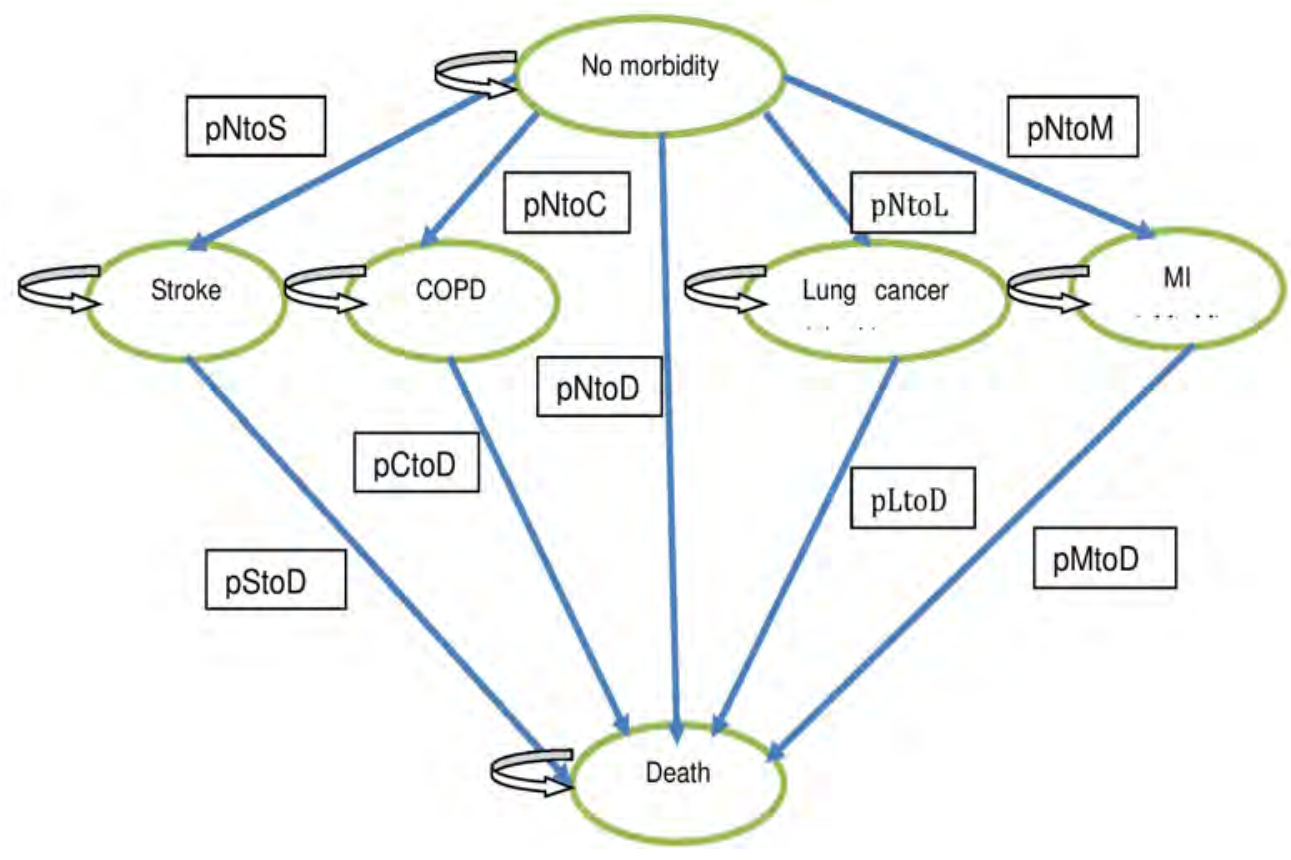

Figure 3. Markov modelfor cost-utility analysis of Veranicline versus Bupropion

Overall, there are six health states on this Markov model. Patients start the model with "well" states, or without morbidity, and can move to particular states such as stroke, COPD, lung cancer and MI or death. The next stage is incorporating input parameters that include transition probability, costs, utility and effectiveness. For this model, the effectiveness of intervention is taken from meta analyses, represented with Risk Ratio (RR).

According to the graph, transition probability initiated with "p" followed by the first letter of states. For example: pStoD is for transition probability from stroke state to death state. Transition probabilities (represented by arrows) are derived from clinical trials, observational studies or from systematic reviews. Transition probabilities can be structured by transition probability matrix on Table 2. The "loop" symbol means that patients may remain experiencing with health condition in that state at specific time before moving to another state. In CUA, sometimes an ideal model is added with second year of progressive states such as: post stroke, post MI, second year lung cancer 
and soon, with an assumption that progressivity of utility values occur in patients after receiving the intervention. If the model having extended structure such as post-stroke or post MI, it means that we could add new input parameters for second year onwards.

Table 2. Transition Probability Matrix

\begin{tabular}{lllllll}
\hline \multirow{2}{*}{$\begin{array}{l}\text { Transition } \\
\text { from }\end{array}$} & No morbidity & Stroke & COPD & Lung cancer & MI & Death \\
\cline { 2 - 7 } $\begin{array}{l}\text { No morbid- } \\
\text { ity }\end{array}$ & $\begin{array}{l}\text { 1-pNtoS-pNtoC- } \\
\text { pNtoL-pNtoMI- } \\
\text { pNtoD }\end{array}$ & pNtoS & pNtoC & pNtoL & pNtoMI & pNtoD \\
\hline Stroke & 0 & $1-$ pstoD & 0 & 0 & 0 & pStoD \\
\hline COPD & 0 & 0 & $1-$ pCtoD & 0 & 0 & pCtoD \\
\hline Lung cancer & 0 & 0 & 0 & $1-$ pLtoD & 0 & pLtoD \\
\hline MI & 0 & 0 & 0 & 0 & 1 -ptoD & ptoD \\
\hline Death & 0 & 0 & 0 & 0 & 0 & pDtoD \\
\hline
\end{tabular}

The total sum of transition probabilities from initial until end of model must be 1.0. Therefore, when we calculate the probability of patients who remain in the same states (loop symbol) with 1-probabilties. Value " 0 " simply representing that there is no transition probability from a state to another state (see figure 2.)

Sometimes, when it is intended to input parameters such as transition probabilities, for instance from systematic reviews, the confusion between "rate" and "probability" exists. Transition probabilities gathered from literature reviews do not exactly reflect the model's cycle that we construct (Sato, 2010). Rates are potential occurrence of an event in defined population and defined time. Meanwhile, probability is assigned values ranging from 0 and 1, representing the "likelihood of an event happening over a specific period of time". To deal with this, rate is possible to be converted to a probability and vice versa (Briggs et al. 2006) where $p$ is the probability, $r$ is the rate and $t$ is the time period of an interest:

\section{$p=1-\exp \{-\mathbf{r t}\}$}

In the cohort hypothetical simulation, the author desribes how Markov model is running, without assigning all input parameters. Details are provided in appendix 1 . We only incorporated transition probability for every health states, only for familiarizing audience of basic Markov application. Unlike a decision tree, Markov model is applicable for applying discounting, which is an important factor in health economic evaluation. Additionally, for chronic diseases, the life table and survival data are often attached into the model. Quality Adjusted Life Years (QALYs) also can be estimated as final results, since in calculating QALYs, valuing quality of life encompasses the length of time spent in health states (Briggs, 1998). The cost analysis also has similar method, costs are represented in treatment events. With computer application, this complex simulation can be handled. After simulating decision analytic model, the final results are ICER, derived from differences for both costs and effects/utility, in this case after 20 years cycle. Thus, decision rules should be followed. 
Although Markov model application is helpful in decision analytic modeling, there are several limitations that we should aware of. Markov has a characteristic of "memory less" (Sonnenberg, 1993; Briggs, 1998). It means when patient move to another states, the model will have no historical memory where patients has come from previous cycles. The population equal in this model also with constant risk. Adding additional states and time dependency into transition probability may possible for the model. Besides, Markov model has greater complexity compared to decision tree, however computer software nowadays could help for running this model.

\section{Handling uncertainty}

Variability, uncertainty and heterogeneity influences the results of decision analytic model, these should be handled (Barton et al. 2004). The results of evaluation are depending on uncertainties that related with various factors. This section will focus on short-review for handling uncertainty, before going further, that will be useful to define several types of uncertainty in modeling (Briggs et al. 2012). Decision makers needs evidence beyond point estimate of the outcome. The uncertainty-surrounding outcome should be explored and reported. Uncertainty may categorized as methodological uncertainty, parameter uncertainty, structural uncertainty and generalizability (Briggs and Sclupher, 1995). Thus, decision maker can receive more information what factors that influence the model and its findings, and increasing the confidence level where they read and intend to accept the evaluation results.

Uncertainty can be handled by performing appropriate sensitivity analysis. There are several types of sensitivity analysis (Andronis et al. 2009, Briggs et al. 2012). First, a one-way sensitivity analysis. It is the simplest form of sensitivity analysis, examines impact of the changes in model results by varying plausible values range. Tornado diagram is used to visualized this, presented with baseline value and range variation in the right and left wings. Second is multi-way sensitivity analysis, including two-way sensitivity analysis. This examines relationship of two or more parameters, not single parameters like one-way sensitivity analyses. Another type is extreme scenario, simply when the parameters or input value of favorable intervention are established to provide best and worst case scenario.

Probabilistic Sensitivity Analysis (PSA) is one type of sensitivity analysis that runs randomly by large number of Monte Carlo simulation (for instance: 10,000 iterative). It is examine each input parameters with their probability distributions. It resulted mean cost as well as effectiveness. This approach is preferred to generate cost-effectiveness acceptability curve (Claxton et al. 2005; Ades et al. 2006).

Finally, model result and its sensitivity analysis should be presented clearly. It is advisable to see guidelines related to full health economics study reporting (Husereau et al. 2013).

\section{Conclusions}

Models are chosen, construct and developed based on decision problems and must be appropriate with decision makers' purpose. All evidence as input parameters are assigned and performs by mathematical operations that translating into model. Furthermore, to decide the set of alternatives that should be chosen, the final results of decision analytical models have to follow decision rules for cost-effectiveness of health intervention. The explanation in this paper not providing best practice yet simple example of modeling application, further details and international guidelines are available elsewhere (Sclupher et al. 2000; Hjelmegren et al. 2001; Weinstein et al. 
2003; Philips et al. 2006).

This paper provides a review and application of decision analytic model in economic evaluation, with tobacco cessation examples. Decision tree and Markov model are commonly performed in health economic evaluation, indeed with their advantages and limitations. These two model are complements each other. Performing decision analytic modeling is beneficial, specifically when there are several barriers from clinical trials. Decision tree with its simplicity is able to provide clear pathways of decision and for economic evaluation with intermediate outcomes. Markov meanwhile has ability to analyze the condition with longer time horizon and expecting final outcomes.

\section{Acknowledgements}

The author thanks to Prof. Hasbullah Thabrany MD.,MPH.,DrPH for his valuable input and review on final draft of manuscript.

\section{References}

Ades, A.E., Claxton, K. and Sculpher, M., 2006. Evidence synthesis, parameter correlation and probabilistic sensitivity analysis. Health economics, 15(4), pp.373-381.

Andronis, L., Barton, P. and Bryan, S., 2009. Sensitivity analysis in economic evaluation: an audit of NICE current practice and a review of its use and value in decision-making.

Bauld, L., Boyd, K.A., Briggs, A.H., Chesterman, J., Ferguson, J., Judge, K. and Hiscock, R., 2011. One-year outcomes and a cost-effectiveness analysis for smokers accessing group-based and pharmacy-led cessation services. Nicotine \& Tobacco Research, 13(2), pp.135-145.

Bolin, K., Wilson, K., Benhaddi, H., De Nigris, E., Marbaix, S., Mork, A.C. and Aubin, H.J., 2009. Cost-effectiveness of varenicline compared with nicotine patches for smoking cessation - results from four European countries. The European Journal of Public Health, 19(6), pp.650-654.

Briggs, A. and Sculpher, M., 1995. Sensitivity analysis in economic evaluation: a review of published studies. Health economics, 4(5), pp.355-371.

Briggs, M.A. and Sculpher, M., 1998. An introduction to Markov modeling for economic evaluation. Pharmacoeconomics, 13(4), pp.397-409.

Briggs, A. and Tambour, M., 2001. The design and analysis of stochastic cost-effectiveness studies for the evaluation of health care interventions.Drug information journal, 35(4), pp.14551468 .

Briggs, A., Sculpher, M. and Claxton, K., 2006. Decision modeling for health economic evaluation. OUP Oxford.

Briggs, A.H., Weinstein, M.C., Fenwick, E.A., Karnon, J., Sculpher, M.J. and Paltiel, A.D., 2012. Model parameter estimation and uncertainty analysis a report of the ISPOR-SMDM Modeling Good Research Practices Task Force Working Group-6. Medical Decision Making, 32(5), pp.722-732.

Buxton, M.J., Drummond, M.F., Van Hout, B.A., Prince, R.L., Sheldon, T.A., Szucs, T. and Vray, M., 1997. Modeling in economic evaluation: an unavoidable fact of life. Health economics, 6(3), pp.217-227.

Byford, S. and Raftery, J., 1998. Economics notes: Perspectives in economic evaluation. British 
Medical Journal, 316(7143), p.1529.

Caro, J.J., Briggs, A.H., Siebert, U. and Kuntz, K.M., 2012. Modeling good research practicesoverview a report of the ISPOR-SMDM modeling good research practices task force-1. Medical Decision Making, 32(5), pp.667-677.

Claxton, K., Sculpher, M., McCabe, C., Briggs, A., Akehurst, R., Buxton, M., Brazier, J. and O’Hagan, T., 2005. Probabilistic sensitivity analysis for NICE technology assessment: not an optional extra. Health economics, 14(4), pp.339-347

Cohen, D.J. and Reynolds, M.R., 2008. Interpreting the results of cost-effectiveness studies. Journal of the American College of Cardiology, 52(25), pp.2119-2126.

Drummond MF, Sculpher MJ, Torrance GW, O’Brien BJ, Stoddart G. 2005. Methods for theeconomic evaluation of health care programmes. 3rd ed. Oxford UniversityPress.

Gray, A.M., Clarke, P.M., Wolstenholme, J.L. and Wordsworth, S., 2010. Applied methods of cost-effectiveness analysis in healthcare (Vol. 3). OUP Oxford.

Grosse SD. Assessing cost-effectiveness in healthcare: history of the $\$ 50,000$ per QALY threshold. Expert review of pharmacoeconomics \& outcomes research. 2008 Apr 1;8(2):165-78.

Hjelmgren, J., Berggren, F. and Andersson, F., 2001. Health economic guidelines - similarities, differences and some implications. Value in Health,4(3), pp.225-250.

Hunnink,M. and P.Glasziou., 2001. Decision Making in Health and Medicine. Cambridge University Press, Cambridge, UK.

Hurley, S.F. and Matthews, J.P., 2007. The Quit Benefits Model: a Markov model for assessing the health benefits and health care cost savings of quitting smoking. Cost effectiveness and resource allocation, 5(1), p.1.

Husereau, D., Drummond, M., Petrou, S., Carswell, C., Moher, D., Greenberg, D., Augustovski, F., Briggs, A.H., Mauskopf, J., Loder, E. and ISPOR Health Economic Evaluation Publication Guidelines-CHEERS Good Reporting Practices Task Force, 2013. Consolidated health economic evaluation reporting standards (CHEERS) — explanation and elaboration: a report of the ISPOR health economic evaluation publication guidelines good reporting practices task force. Value in Health, 16(2), pp.231-250. Jefferson, T., Demicheli, V. and Mugford, M., 2000. Elementary economic evaluation in health care. Wiley-Blackwell [Imprint].

Jönsson, B., 2009. Ten arguments for a societal perspective in the economic evaluation of medical innovations. The European Journal of Health Economics, 10(4), pp.357-359.

Karnon, J. and Brown, J., 1998. Selecting a decision model for economic evaluation: a case study and review. Health care management science, 1(2), pp.133-140.

Martikainen, J., 2008. Application of decision-analytic modeling in health economic evaluations. University of Kuopio.

McCabe, C., Claxton, K. and Culyer, A.J., 2008. The NICE cost-effectiveness threshold. Pharmacoeconomics, 26(9), pp.733-744.

Morris,S., N. Devlin, and D. Parkin., 2007. Economic Analysis in Health Care. John Wiley \& Sons, Chichester, UK.

Neumann, P.J., Cohen, J.T. and Weinstein, M.C., 2014. Updating cost-effectiveness - the curious resilience of the \$50,000-per-QALY threshold. New England Journal of Medicine, 371(9), pp.796-797.

O’Brien, B.J., Drummond, M.F., Labelle, R.J. and Willan, A., 1994. In search of power and signif- 
icance: issues in the design and analysis of stochastic cost-effectiveness studies in health care. Medical care, pp.150-163.

Owens, D.K., 1998. Interpretation of cost-effectiveness analyses. Journal of General Internal Medicine, 13(10), p.716.

Petitti, D.B., 1999. Meta-analysis, decision analysis, and cost-effectiveness analysis: methods for quantitative synthesis in medicine. Oxford University Press.

Petrou, S. and Gray, A., 2011. Economic evaluation using decision analytical modeling: design, conduct, analysis, and reporting. Bmj, 342, p.d1766.

Philips, Z., Bojke, L., Sculpher, M., Claxton, K. and Golder, S., 2006. Good practice guidelines for decision-analytic modeling in health technology assessment. Pharmacoeconomics, 24(4), pp.355-371.

Roberts, M., Russell, L.B., Paltiel, A.D., Chambers, M., McEwan, P. and Krahn, M., 2012. Conceptualizing a model a report of the ISPOR-SMDM modeling good research practices task force-2. Medical Decision Making, 32(5), pp.678-689.

Ruger, J.P. and Lazar, C.M., 2012. Economic evaluation of pharmacy-and behavioral therapies for smoking cessation: a critical and systematic review of empirical research. Annual review of public health, 33, p.279.

Sato, R.C. and Zouain, D.M., 2010. Markov Models in health care. Einstein (São Paulo), 8(3), pp.376-379.

Sculpher, M., Drummond, M. and Buxton, M., 1997. The iterative use of economic evaluation as part of the process of health technology assessment. Journal of Health Services Research, 2(1), pp.26-30.

Sculpher, M., Fenwick, E. and Claxton, K., 2000. Assessing quality in decision analytic cost-effectiveness models. Pharmacoeconomics, 17(5), pp.461-477.

Sculpher, M.J., Claxton, K., Drummond, M. and McCabe, C., 2006. Whither trial based economic evaluations for health caredecision-making?. Health economics, 15(7), pp.677-687.

Shiell, A., Donaldson, C., Mitton, C. and Currie, G., 2002. Health economic evaluation. Journal of epidemiology and community health, 56(2), p.85.

Shiroiwa, T., Sung, Y.K., Fukuda, T., Lang, H.C., Bae, S.C. and Tsutani, K., 2010. International survey on willingness to pay (WTP) for one additional QALY gained: what is the threshold of cost-effectiveness?. Health economics, 19(4), pp.422-437.

Sonnenberg, F.A. and Beck, J.R., 1993. Markov models in medical decision making a practical guide. Medical decision making, 13(4), pp.322-338

Sox,H.C., Blatt, M,A., Higgins,M.C., and Marton, K.I. 1988.Medical Decision Making.Butterworths, Stoneham, Massachusetts.

Stahl, J.E., 2008. Modeling methods for pharmacoeconomics and health technology assessment. Pharmacoeconomics, 26(2), pp.131-148.

Tran, M.T., Holdford, D.A., Kennedy, D.T. and Small, R.E., 2002. Modeling the Cost Effectiveness of a Smoking $\square$ Cessation Program in a Community Pharmacy Practice. Pharmacotherapy: The Journal of Human Pharmacology and Drug Therapy, 22(12), pp.1623-1631.

Weinstein, M.C., O’Brien, B., Hornberger, J., Jackson, J., Johannesson, M., McCabe, C. and Luce, B.R., 2003. Principles of good practice for decision analytic modeling in health care evaluation: report of the ISPOR Task Force on Good Research Practices-Modeling Studies. Value in health, 6(1), pp.9-17. 


\section{Appendix 1}

Table 1. Input Parameters

\begin{tabular}{cc}
\hline \multicolumn{1}{c}{ Parameters } & Value \\
\hline Transition probabilities & \\
pNtoS & 0.0082 \\
pNtoC & 0.0721 \\
pNtoL & 0.040 \\
pNtoM & 0.01 \\
pNtoD & 0.05 \\
pStoD & 0.022 \\
pCtoD & 0.025 \\
pLtoD & 0.075 \\
pMtoD & 0.035 \\
pDtoD & 1.00 \\
Effectiveness & \\
RRs & 0.71 \\
RRc & 0.65 \\
RRl & 0.85 \\
RRm & 0.69 \\
\hline
\end{tabular}




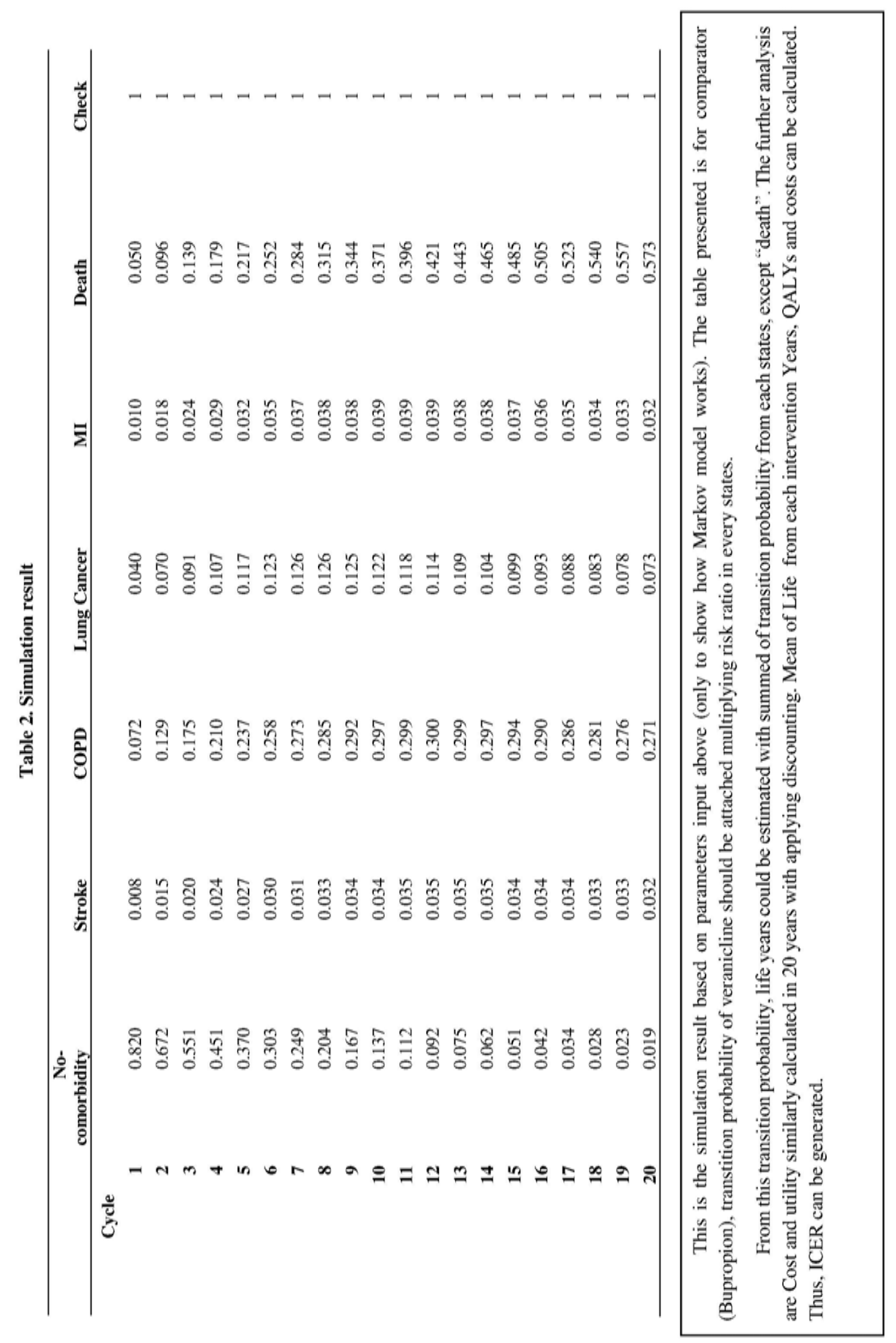

